\title{
Measurements of Elastic Properties of Tendons: Comparison of Two Approaches
}

\author{
Po-Ling Kuo and Pai-Chi Li* \\ Department of Physical Medicine and Rehabilitation, National Taiwan University Hospital \\ *Department of Electrical Engineering, National Taiwan University, \\ Taipei, Taiwan, R. O. C
}

Abstract- Ultrasonic elasticity imaging has great potential in rehabilitative medicine due to its ability to quantitatively measure mechanical properties of tendon. In this paper, two different approaches for assessing elastic properties of tendon were employed. Six bovine Achilles tendon specimens were used. The first approach directly measured Young's moduli along the transverse direction (i.e., $E_{\text {perpendicular }}$ ) and the longitudinal direction (i.e., $E_{\text {parallel }}$ ) using an electronic balance, a compressor positioned by a 3 -axis stepping motor system and a personal computer for central control and data acquisition. Young's moduli were derived based on the measured strain and stress values. The second approach was based on the assumption that tendons are transversely isotropic. Three observable second-order elastic stiffness constants $\left(c_{11}, c_{13}\right.$ and $\left.c_{33}\right)$ were obtained by measuring the speed of sound along different directions of propagation using a transmission mode method. Sound velocity along 0, 30, 45 and 90 degrees relative to the fiber axis were measured for all six specimens. The measured elastic stiffness constants were also compared to the average $E_{\text {perpendicular }}$ and $E_{\text {parallel }}$ obtained from the first set of experiments. It was found that measurements in first approach were influenced by viscoelasticity, thus resulting in significant discrepancy between the two approaches. In this paper, details of the two approaches are presented. Potential sources of the discrepancy are also discussed.

\section{INTRODUCTION}

Tendon dysfunction is a major issue in rehabilitative medicine. Because of its potential ability to quantitatively measure mechanical properties of tissue, ultrasonic elasticity imaging may be of great use to monitor the tendon functions during rehabilitation process.
We have previously shown that strain imaging may be a better tool for clinical assessment of tendon inflammation than traditional B-mode sonogram [1]. Given the strain fields, the next step is to reconstruct elastic properties of tendon. Therefore, knowledge of distribution of elastic modulus in tendon is crucial. Specifically, values of Young's modulus along the longitudinal fiber axis (i.e., $\mathrm{E}_{\text {paraliel }}$ ) and the direction perpendicular to the fiber axis (i.e., $E_{\text {perpendicular }}$ ), are of great importance to describe the mechanical properties of tendon.

Mechanical properties of tendon have been obtained using the transversely isotropic model [2-4]. The Young's modulus, however, was derived from observable elastic stiffness coefficients without correlating with direct measurements [4]. Furthermore, the data were obtained from formalin fixed samples, which had mechanical properties different from those in living tissue. To address these issues, we employ two different approaches to assess elastic properties of fresh tendon samples. The first approach is to directly measure Young's modulus parallel and perpendicular to the longitudinal fiber axis. Another approach uses coefficients of elastic stiffness tensor to determine values of Young's modulus based on the assumption that tendons are transversely isotropic. Data from both approaches are correlated and potential sources of discrepancy are discussed.

\section{THEORY}

Elastic properties of tendon can be described by an elastic tensor with hexagonal symmetry. A hexagonal system requires five independent elastic stiffness coefficients to describe its stress-strain relationship, i.e., $c_{11}, c_{13}, c_{33}, c_{44}$ and $c_{66}$ by the Voigt notation [4]. Note that the fiber axis is along the 3 -axis. Based on this model, the values of $c_{33}$ and $c_{11}$ can be expressed as 


$$
c_{11}=\rho V_{\text {perpendictlar }}^{2}
$$

and

$$
c_{33}=\rho V_{\text {purallel }}^{2}
$$

where $\rho$ denotes the density of tendon [2]. $V_{\text {parallel }}$ and $V_{\text {perpendicular }}$ represent the sound velocity parallel and perpendicular to the fiber axis, respectively. The phase velocity of longitudinal wave propagating at any direction in the plane containing the fiber axis can be described by the equation

$$
\begin{aligned}
V_{L}= & \left(\left\{\left(c_{11} \sin ^{2} \theta+c_{33} \cos ^{2} \theta+c_{44}\right)\right.\right. \\
+ & \left(\left[\left(c_{11}-c_{44}\right) \sin ^{2} \theta-\left(c_{33}-c_{44}\right) \cos ^{2} \theta\right]^{2}\right. \\
& \left.\left.\left.+4\left(c_{13}+c_{44}\right)^{2} \sin ^{2} \theta \cos ^{2} \theta\right)^{1 / 2}\right\}^{1 / 2}\right)(2 \rho)^{-1 / 2}
\end{aligned}
$$

where $\theta$ denotes the angle relative to the fiber axis [3]. Thus, the elastic stiffness coefficient $c_{13}$ can also be obtained based on equation (3). In other words,

$$
\begin{aligned}
c_{13}= & \left\{\left[\left(c_{33} \cos ^{2} \theta+c_{44} \sin ^{2} \theta\right)\left(c_{11} \sin ^{2} \theta+c_{44} \cos ^{2} \theta\right)\right.\right. \\
& \left.\left.-\left(c_{11} \sin ^{2} \theta+c_{33} \cos ^{2} \theta+c_{44}\right) p V_{L}^{2}+\rho^{2} V_{L}^{4}\right]^{1 / 2}\right\} \\
& \times(|\sin \theta \| \cos \theta|)^{-1}-c_{44} .
\end{aligned}
$$

The Young's modulus, defined as the ratio of the loading stress to the corresponding strain, can also be derived from coefficients of the elastic stiffness tensor. Specifically, the Young's moduli perpendicular and parallel to the fiber axis can be expresses as

$$
E_{\text {parallet }}=c_{33}-\frac{c_{13}^{2}}{c_{11}-c_{66}}
$$

and

$$
E_{\text {perpendicular }}=2 c_{66}\left[2-\frac{2 c_{33} c_{66}}{c_{11} c_{33}-c_{13}^{2}}\right] .
$$

Equations (5) and (6) show that four independent elastic stiffness coefficients $\left(c_{11}, c_{33}, c_{13}\right.$ and $\left.c_{66}\right)$ are needed to determine $E_{\text {perpendicular }}$ and $E_{\text {parallel. }}$.

\section{MATERIALS AND METHODS}

Samples used in this study were prepared from fresh specimens of six bovine deep flexor tendons from the Achilles group. For each tendon, a cubic specimen and two discoid specimens were prepared and preserved in a normal saline solution for future use. The cubic specimen was cut for measuring sound speeds parallel and perpendicular to the fiber axis. The same specimen was also used for direct measurements of Young's modulus along the same direction. The two discoid specimens were obtained by two parallel cuts at 30 and 45 degrees relative to the longitudinal axis. Speeds of sound at four different propagating directions $(0,30,45$ and 90 degrees relative to the longitudinal fiber axis) were measured for each tendon.

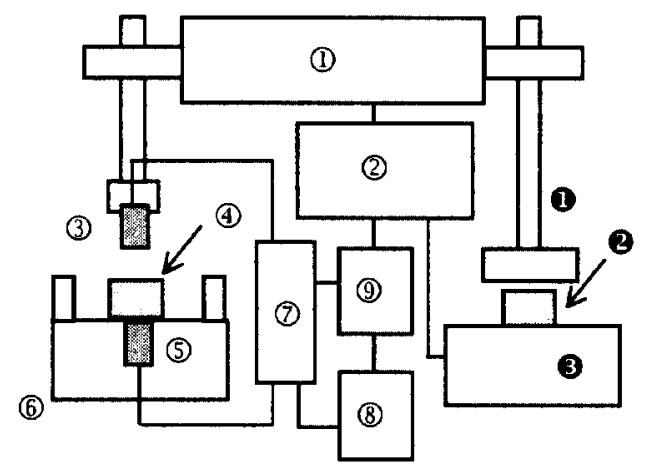

Fig. 1

As shown in Fig. 1, the experimental setup using transmission mode for velocity measurements included a transmitter (3) (Panametrics V310,5-MHz) and a receiver (5) (Panametrics V383, 3.5-MHz). A custom made acrylic holder (6) with an acrylic enclosure was used to secure the receiver and hold water between the two transducers. Ultrasonic pulses were generated and received by a commercial pulser-receiver (Panametrics 5072PR) (8). A tendon sample (4) was first placed on the center of receiving transducer and the transmitting transducer was moved toward the upper surface of the specimen using the step motor system (1). A digital oscilloscope (2) (LeCroy 9304AM) was used to determine the optimal pulser-receiver settings. The ultrasonic signal was digitized at a sampling rate of $20-\mathrm{MHz}$ by an A/D converter (8) and stored in a personal computer (2) for off-line analysis. After the measurement was complete, the sample was removed without changing the position of the transducers and water was filled into the acrylic holder between the two transducers. Another ultrasonic measurement was done to determine the length of sample given the speed of sound in water.

The mass density, defined as ratio of weight to volume, was determined by small samples removed from the tendons. For each sample, weight was determined by an electronic balance with a readability of $0.1 \mathrm{~g}$ (OHAUS IP12KS), and volume was measured by a measuring glass with the minimum 
scale of $0.5 \mathrm{mI}$.

For each tendon, $E_{\text {perpendicular }}$ and $E_{\text {parallel }}$ were obtained by using the setup shown in Fig. 1. After putting each cubic specimen 2 on the center of the electronic balance 3 with its longitudinal fiber axis parallel or perpendicular to the surface of the balance, the acrylic compressor was carefully positioned by the step motor system (1) toward the sample. Note that original height of each sample was determined beforehand using water measurements. The sample was then cyclically compressed at a $9.1-\mathrm{step} / \mathrm{s}$ rate with $5-\mu \mathrm{m}$ advancement in each step. Readings of the electronic balance from five consecutive compression -relaxation cycles were acquired and stored into a personal computer (2) via a RS232 port. The area of tissue surface between the sample and electronic balance was measured to calculate stress.

The sound velocity in tendon along a specific direction was derived from the equation

$$
V_{\text {ussue }}=V_{\text {water }} \times \frac{T_{\text {watter }}}{T_{\text {ussue }}},
$$

where $V_{\text {waer }}$ represents the velocity of sound in water at room temperature, $T_{\text {susue }}$ and $T_{\text {wuter }}$ denote the times of flight between transducers with tissue and water in between the two transducers, respectively. For each recording, the time of flight was measured by detecting the time when the RF signal first exceeded a pre-specified threshold. For all measurements, the threshold was 0.001 out of a 0.21 signal range.

The elastic stiffness coefficients $c_{33}$ and $c_{11}$ were obtained according to equations (1) and (2), respectively. The elastic stiffness coefficient $c_{13}$ was determined by the following procedures. First, sound velocity measured at the four angles was used to interpolate the velocity at other angles based on the assumption that tendon is transversely isotropic. As shown in Fig. 2, a velocity curve as a function of angle was obtained using spline interpolation. Note that the velocity curve is approximately linear between 30 and 45 degrees. Thus by using a 1 -degree increment, there are totally 16 values that can be used to generate 16 corresponding $c_{13}$ estimates based on equation (4). The value of $c_{44}$, which corresponds to the propagation of transverse mode ultrasonic waves through the tissue, was initially set to zero [2-3]. The value of $c_{44}$ was then varied over several orders of magnitude to check its effects on $c_{13}$ estimation and we found that the change in $c_{13}$ due to different $c_{44}$ was less than $1 \%$. Next, each estimated $c_{13}$ was applied to equation (3) with the angle varying from 30 to 45 degrees. Totally 256 (i.e., 16 times 16) velocity estimates were obtained. The $c_{13}$ value with the minimal mean squared errors between the estimated velocity values and the interpolated curve was selected as the optimal $c_{13}$ estimate.

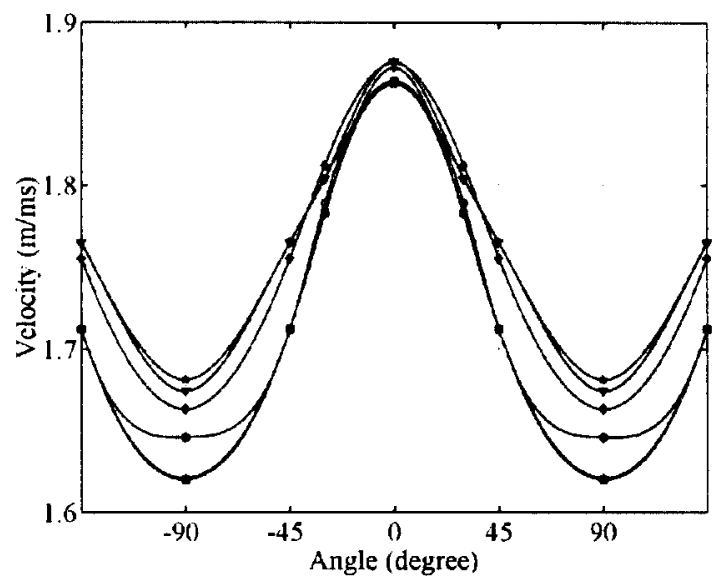

Fig. 2

$E_{\text {perpendicular }}$ and $E_{\text {parallel }}$ in each tendon were computed from the slope of stress-strain curve. The strain for each measurement was determined by the ratio of total deformation to the initial height. Since the compressor was position by the computer controlled step motor, the strain can be readily obtained. The stress was obtained by dividing the reading of the electronic balance by the area of contacting surface between the sample and the balance. Finally, a best-fit linear curve was used. Since the two initial compression-decompression cycles were used for preloading the tendon, the corresponding data were not included for estimation [5]. Note that the linear coefficients in the last three cycles were very similar to each other and the differences among them were less than 1-Kpa. Hence the mean value was chosen as the estimated Young's modulus.

Finally, the measured $E_{\text {parallel }}$ was used in equation (5) along with $c_{11}, c_{33}$ and $c_{13}$ to estimate $c_{60}$ for each tendon. The estimated $c_{66}$ was then used in equation (6) to estimate $E_{\text {perpendicular. The results were }}$ later correlated with the actual measurements.

\section{RESULTS}

In Table 1, results of mass density and velocity measurements for six tendons are summarized, where $\rho$ represents the density, $V_{0}$ denotes the sound 
velocity propagating along the fiber axis, $V_{90}$ represents the velocity perpendicular to fiber axis, $V_{30}$ and $V_{45}$ denote the velocity 30 and 45 degrees relative to the fiber axis, respectively. A mong the six tendons, results of velocity measurement compared well to one another and velocities of all six tendons have the same gross dependency on angle.

Table 1.

\begin{tabular}{llllll}
\hline Sample & $\rho(\mathrm{g} / \mathrm{cm} 3)$ & $V_{0}(\mathrm{~m} / \mathrm{s})$ & $V_{30}(\mathrm{~m} / \mathrm{s})$ & $V_{45}(\mathrm{~m} / \mathrm{s})$ & $V_{90}(\mathrm{~m} / \mathrm{s})$ \\
\hline 1 & 1.10 & 1864 & 1783 & 1712 & 1621 \\
2 & 1.09 & 1864 & 1789 & 1712 & 1638 \\
3 & 1.10 & 1864 & 1789 & 1712 & 1646 \\
4 & 1.10 & 1858 & 1804 & 1765 & 1663 \\
5 & 1.09 & 1854 & 1804 & 1755 & 1674 \\
6 & 1.11 & 1862 & 1812 & 1765 & 1681 \\
Average & 1.10 & 1861 & 1797 & 1767 & 1654 \\
\hline
\end{tabular}

Tables 2 and 3 list results of estimated elastic stiffness coefficients, measured Young's moduli and estimated Young's moduli. Again, angle dependency of elastic stiffness coefficients was clearly visible.

Table 2 .

\begin{tabular}{lllll}
\hline Sample & $C_{11}(\mathrm{Gpa})$ & $\mathrm{C}_{33}(\mathrm{Gpa})$ & $\mathrm{C}_{13}(\mathrm{Gpa})$ & $\mathrm{C}_{66}(\mathrm{Gpa})$ \\
\hline 1 & 2.8904 & 3.8219 & 3.0542 & 0.4496 \\
2 & 2.9513 & 3.8219 & 3.0644 & 0.4941 \\
3 & 2.9802 & 3.8219 & 3.0555 & 0.5373 \\
4 & 3.0421 & 3.7974 & 3.3737 & 0.0447 \\
5 & 3.0825 & 3.7810 & 3.3754 & 0.0691 \\
6 & 3.1083 & 3.81379 & 3.3718 & 0.1271 \\
Average 3.0091 & 3.8096 & 3.2158 & 0.2870 \\
\hline
\end{tabular}

The measured Young's modulus also depended on angle. The estimated values of $c_{66}$ were about an order of magnitude lower than $c_{11}, c_{33}$, or $c_{13}$. Compared with actual measurements, all the six values of Young's modulus estimated from $c_{66}$ (i.e., eE $E_{\text {perpendicular }}$ ) were significantly larger.

Table 3.

\begin{tabular}{lllll}
\hline Sample & $\begin{array}{l}E_{\text {parallel }} \\
(\mathrm{K} p a)\end{array}$ & $\begin{array}{l}E_{\text {perpendicular }} \\
(\mathrm{Kpa})\end{array}$ & $\begin{array}{l}\mathrm{E}_{\text {parallel }} / \\
\mathrm{E}_{\text {perpendicular }}\end{array}$ & $\begin{array}{l}\mathrm{eE}_{\text {perpendicular }} \\
(\mathrm{K} \text { pa })\end{array}$ \\
\hline 1 & 106.1 & 88.9 & 1.19 & 270 \\
2 & 154.3 & 104.2 & 1.48 & 396.7 \\
3 & 104.9 & 84.1 & 1.25 & 268.1 \\
4 & 123.1 & 59.5 & 2.07 & 387.8 \\
5 & 89.1 & 68.7 & 1.29 & 283.7 \\
6 & 115.4 & 80.6 & 1.43 & 360.7 \\
Average & 115.5 & 81.0 & 1.45 & 327.8 \\
\hline
\end{tabular}

\section{DISCUSSION}

Hoffmeister et al. measured elastic stiffness tensor of formalin fixed bovine A chilles tendons [2-4] On the other hand, results of the current paper were based on fresh bovine Achilles tendons. The two studies have good agreement in mass density, anisotropy of velocity and the three elastic stiffness coefficients $c_{11}, c_{33}$ and $c_{13}$ are on the same order of magnitude. On the other hand, the degree of dependency on angle, $V_{0}$, and $c_{33}$ were smaller in our study. Such discrepancy may be explained by the fact that formalin fixation produces a measurable increase in longitudinal-mode ultrasonic velocity [2].

Discrepancy also exists in Young's modulus and $c_{66}$. The increased stiffness due to formalin fixation may increase the Young's modulus found in the literature [4]. Furthermore, a lower strain rate as that used in this study (about $0.005 / \mathrm{s}$ ) may also produce a smaller value for Young's modulus because of the viscoelastic behavior possessed by the tendon [5]. Consequently, the estimated $c_{66}$ may also be affected based on equation (5).

In conclusion, the results reported in this study are based on fresh tendon specimens. Values of mechanical properties from direct measurements were correlated with estimates. Such information is useful in further development work for elasticity imaging of tendons.

\section{REFERENCES}

1. Kuo PL, Li PC, et al. Strain measurements of rabbit Achilles tendons by ultrasound. Ultrasound Med Biol 1999; 25(8): 1241-1250.

2. Hoffmeister BK, Miller JG, et al. Effect of collagen on the anisotropy of quasi-longitudinal mode ultrasonic velocity in fibrous soft tissues: A comparison of fixed tendon and fixed myocardium. J Acoust Soc Am 1994; 96(4): 1957-1964.

3. Hoffmeister BK, Miller JG et al. Estimation of the elastic stiffness coefficient $c_{13}$ of fixed tendon and myocardium. J Acoust Soc Am 1995;97(5): $3171-3176$

4. Hoffmeister BK, Miller JG et al. Ultrasonic determination of the anisotropy of Young's modulus of fixed tendon and fixed myocardium. J Acoust Soc Am 1996; 100(6): 3933-3940.

5. Nordin M, Frankel VH. Basic biomechanics of the musculoskeletal system. Philadelphia: Lea \& Febiger, 1989: 59-71. 